\title{
Scalable linear magneto resistive sensor arrays
}

\author{
Andreas Voss, Axel Bartos \\ TE Sensor Solutions, MEAS Deutschland GmbH, 44227 Dortmund, Germany \\ Andreas.Voss@te.com0231-9740560
}

\section{Summary}

The advancing digitalization of manufacturing ("smart factories") will create a high demand of sensor solutions. Besides environmental monitoring, position sensing will play a key role in future production processes. The magneto resistive (MR) sensor technology provides cost effective and precise solutions for a variety of applications. Especially in pneumatics, short stroke measurements or end point determination are well covered by MR sensor technology due to its very compact size at highly precise detection. Expanding the target applications to hydraulics, level measurement or potentiometer replacement, magneto resistive sensor arrays have the ability to be used in a wide range of applications. Looking to the automotive world, break-pedal sensors or the determination of the clutch position are good examples for the applicability of scalable MR sensor systems, where they are focused to be a potential replacement for the today widely used inductive based PLCD (permanent magnetic linear contactless displacement) technology. In summary, a basic demand from all of the mentioned applications is modularity and scalability to different sensing lengths, environmental specifics like ferromagnetic pistons and spreading accuracy demands. Here we present a magnetic sensor system solution that is easy scalable for the application.

Keywords: magneto resistive, linear measurement, scalable, contactless

\section{Position measurement with anisotropic magneto resistive sensors}

Among the variety of technologies for position sensing, solutions based on the anisotropic magneto resistive (AMR)-effect $[1,2]$ combine high precision and cost efficiency. As the measurement is contactless, AMR based devices work wear free under harsh environments over a wide temperature range.
Its components can also be protected against chemicals and dirt, without affecting the precision of the measurement.

In contrast to Hall sensors, where the position measurement is based on the magnetic field strength, the AMR technology measures the magnetic field direction as shown in figure 1. Although the magnetic field strength could drop due to altering effects [3], the magnetic field direction stays stable over time, which is a big benefit for the use of MR sensor systems.

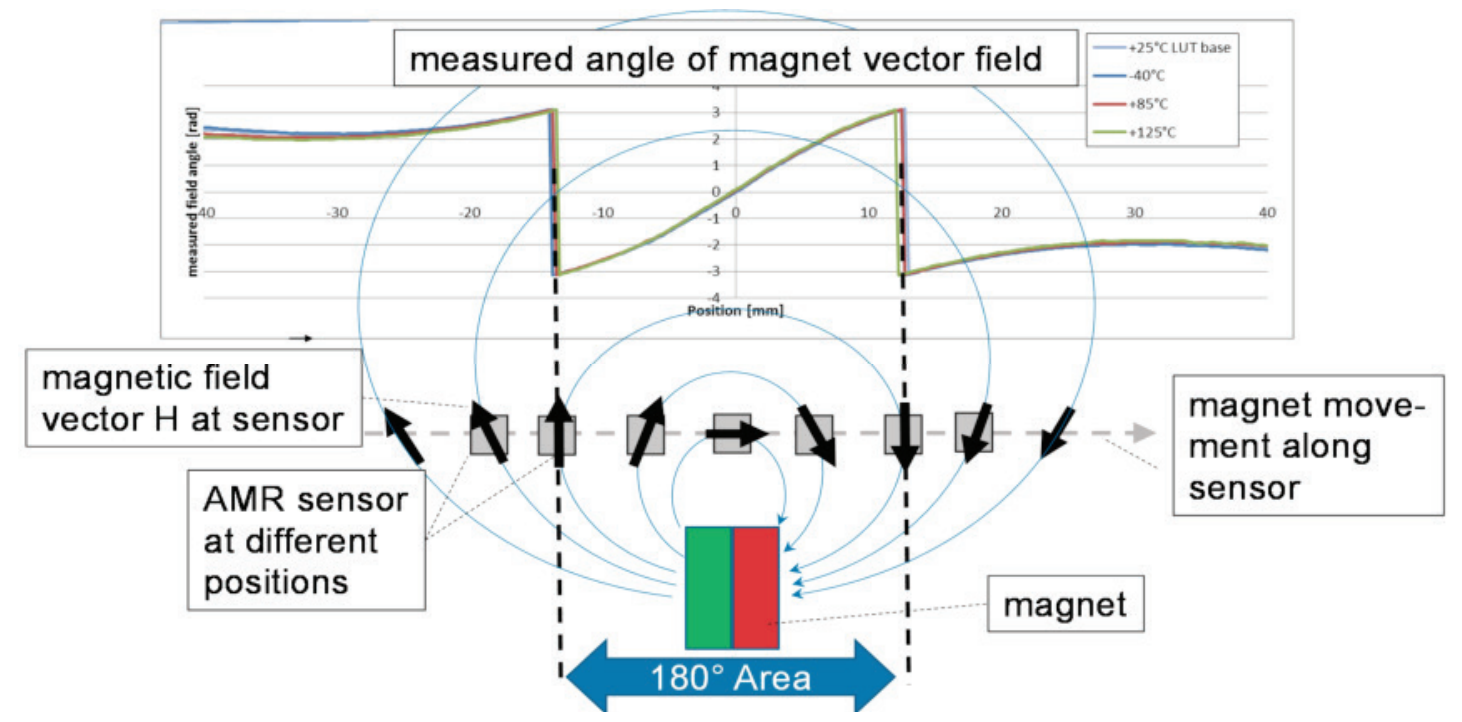

Figure 1 vector distribution $\vec{H}$ of magnetic field when moving a dipole magnet along a MR sensor 
A linear movement of a dipole magnet along the MR sensor generates a unique correlation between magnetic field distribution $\overrightarrow{\mathrm{H}}$ and the position of the magnet,

$x_{\text {pos }}=f_{M R}(\vec{H}(x))$ with $\vec{H}=\left(\begin{array}{c}H_{x} \\ H_{y} \\ H_{z}\end{array}\right)$

where the AMR is only sensitive to a two dimensional projection of the field. To represent the angular information of the measured magnetic vector field, standard magneto resistive angular sensor are used.

\section{AMR angle sensors}

AMR angle sensors are generally made of meandered resistive strips of Permalloy [1], which are produced in thin film technology [4]. Meanders are connected orthogonally to full Wheatstone bridges. The output voltage $U_{x}$ of a sensor bridge depends on the external magnetic field angle $\Theta$ in good approximation by equation (2):

$$
U_{\text {sin }}=U_{o f f, \sin }+f_{\text {gain }} \cdot k_{M R}\left(H_{0}, T\right) \cdot \sin (2 \cdot \Theta)
$$

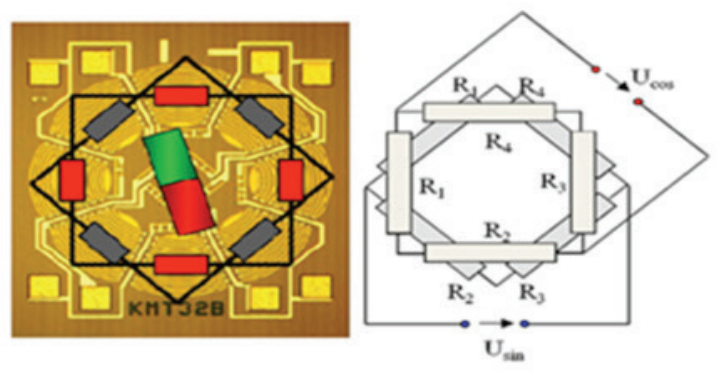

Figure 2 rotating magnet over $A M R$ angle sensor, schematic of the $90^{\circ}$ shifted wheatstone bridges

A standard $180^{\circ}$ AMR rotary angle sensor uses two mutually shifted, nested bridges (Fig. 2 ), to produce the periodic sine and cosine (shown in equation (3) ) output signals, when exposed to a rotational magnetic field.

$$
U_{\text {cos }}=U_{\text {off }, \cos }+f_{\text {gain }} \cdot k_{M R}\left(H_{0}, T\right) \cdot \cos (2 \cdot \Theta)
$$

After cancelling out the offset terms $U_{\text {off } x}$, which are mainly driven by design effects in the analog part of the signal chain (signal line, amplifier and ADC offsets), the offset-free sine and cosine signals can be used to determine an accurate angular information of the measured magnetic field direction.

As seen in Fig. 3 these normal signals are affected mainly by temperature amplitude effects, which are cancelled out by using an arctangent relation as shown in equation (4) and (5):

$$
\begin{aligned}
& U_{\text {sin,offset comp. }}=f_{\text {gain }} \cdot k_{M R}\left(H_{0}, T\right) \cdot \sin (2 \cdot \Theta) \\
& U_{\text {cos,offset comp. }}=f_{\text {gain }} \cdot k_{M R}\left(H_{0}, T\right) \cdot \cos (2 \cdot \Theta) \\
& \Theta=\frac{1}{2} \arctan \left(\frac{f_{\text {gain }} \cdot k_{M R}\left(H_{0}, T\right) \cdot \sin (2 \cdot \Theta)}{f_{\text {gain }} \cdot k_{M R}\left(H_{0}, T\right) \cdot \sin (2 \cdot \Theta)}\right)
\end{aligned}
$$

This leads to a representation of the measured magnetic field angle, free of temperature effects. The resulting angular information remains ambiguous as the AMR-effect does not distinguish between north and south poles and therefore the angular information is only unique in the range of $\left[0 \ldots 180^{\circ}\right]$. Beyond this range the signal repeats periodically.

Moving a dipole magnet along an AMR angle sensor generates sine/cosine signals, which can be used to determine the position of the magnet. As the measured field distribution contains angular changes for more than $180^{\circ}$ and the used AMR angle sensor is just unique for $180^{\circ}$, a periodic output signal is generated as shown in figure $3-\mathrm{C}$.

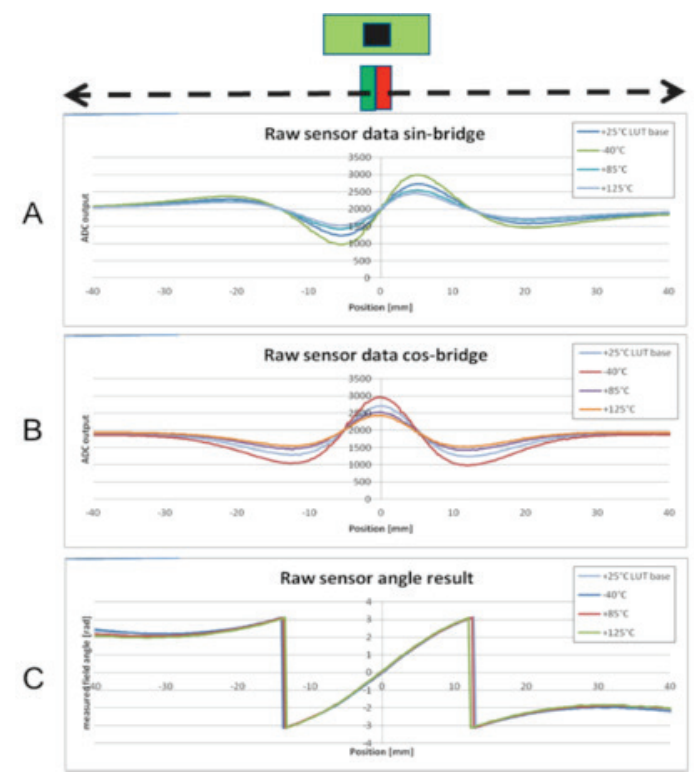

Figure 3 Relationship between sine, cosine and field angle

To filter out the $180^{\circ}$ range of a unique angular determination, the so called amplitude criteria of the used $U_{\sin }$ and $U_{\cos }$ signals is used. Equation (6) is looking to the relationship

$$
r=\sqrt{\left(U_{\sin }\right)^{2}+\left(U_{\cos }\right)^{2}}
$$

which reveals a constant value for $r$ as long as the magnet is next by the sensor. Figure 4 shows this relationship: 


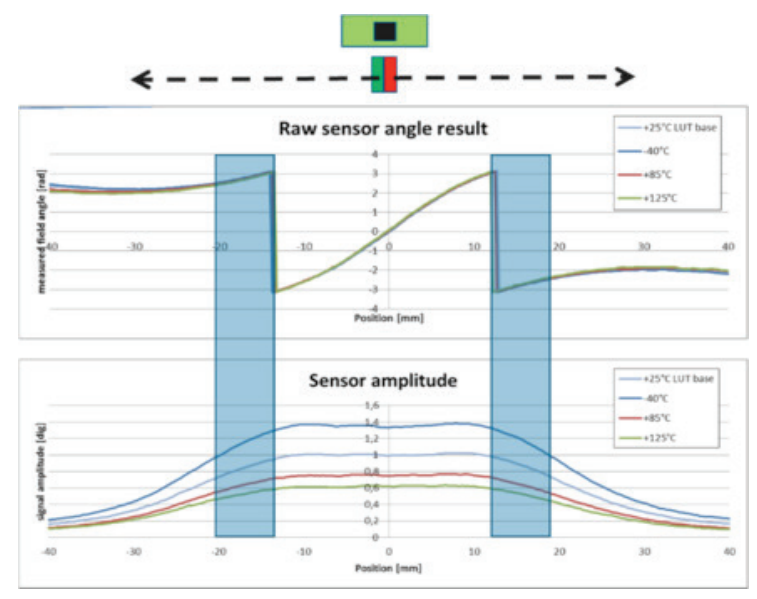

Figure 4 Relationship of measured field angle $\Theta$ and sensor amplitude $r$

This filter criteria is used to restrict the valid output signal of the arctangent calculation to the unique $180^{\circ}$ range, where the magnet is adjacent to the sensor.

\section{Short stroke measurement}

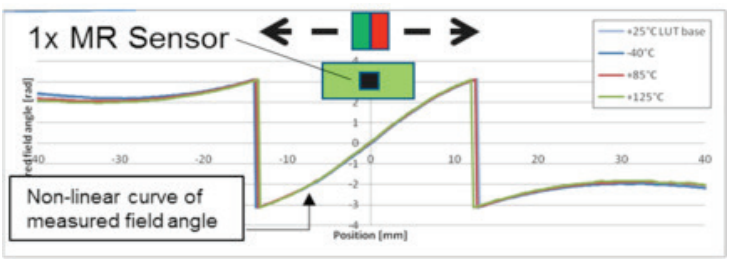

Figure 5 short stroke measurement with one $M R$ sensor, non-linear curve of measured field angle

Assuming a measurement task has to sense the position of an embedded magnet ring inside a pneumatic cylinder as shown in figure 7 , the straight forward approach is to place an angular AMR sensor preferably inside the cylinder. From figure 5 it is seen, that the relationship between measured field angle and moved position of the magnet is not linear. This is explained by the fact, that the AMR angular sensor is originally designed for homogenous vector fields. When bypassing a ring magnet next to an AMR sensor, the measurement is based on an inhomogeneous curved vector field (figure 6) which leads to harmonics in the angle measurement. Therefore the shape of the measured angle curve depends on the following geometrical parameters:

- Shape and geometry of magnet (ring, disk, segment)

- Position of magnet relative to sensor

- Magnetization of magnet

magnet, so it could not be used for longer distances [5].

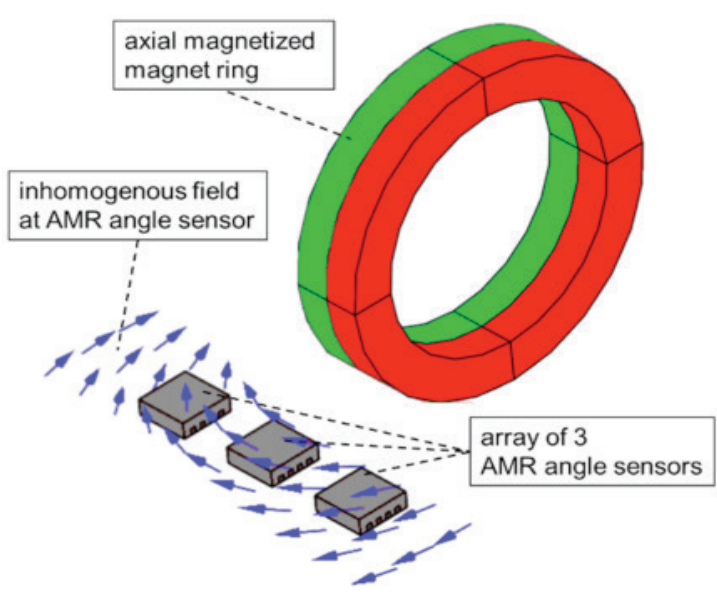

Figure 6 inhomogeneous field distribution of ring magnet at AMR sensor array

To linearize the output function a Look-UpTable (LUT) is used, which results in a linear position mapping, when filtering the angle result at the region of a constant amplitude like described above. This type of measurement is naturally limited by the length of the used

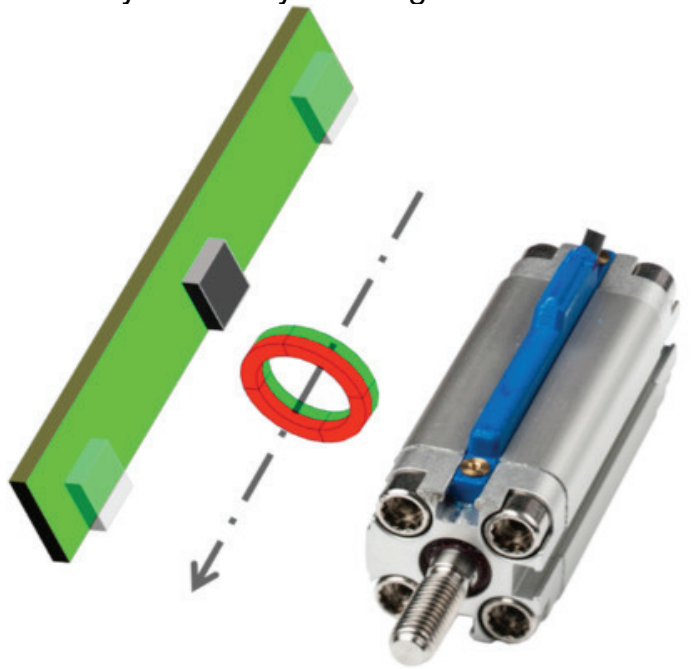

Figure 7 MR sensor embedded in pneumatic cylinder measuring the position of a magnet ring, attached to the cylinders plunger (expansion of measurement range by adding sensors at the edges)

\section{Expansion of measurement range (2 or 3 AMR sensors)}

Coming from short stroke measurements, where just one MR sensor is in place to measure the position of a magnet, the consistently next step is to expand the sensing range by using linear aligned sensor arrays as shown in figure 8: 


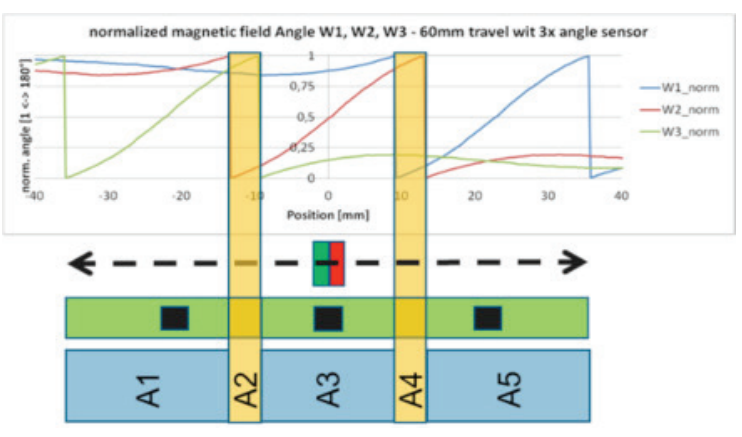

Figure 8 magnet travel along array of $3 A M R$ sensors

This approach needs to deal with a combination of more than one sensor signal to determine the magnet position. A special situation is found when looking to 2 or 3 sensors.

Basically the scanned range is split into several regions (see figure 8: A1 ... A5), where a particular handling by the signal evaluation is needed to discover the correct linear absolute position.

Special attention has to be given to the so called hand-over regions (see figure $8 \mathrm{~A} 2$ and A4), at the range between two adjacent sensors of the chain, where both sensor results could be taken into account, to determine the position of the magnet.

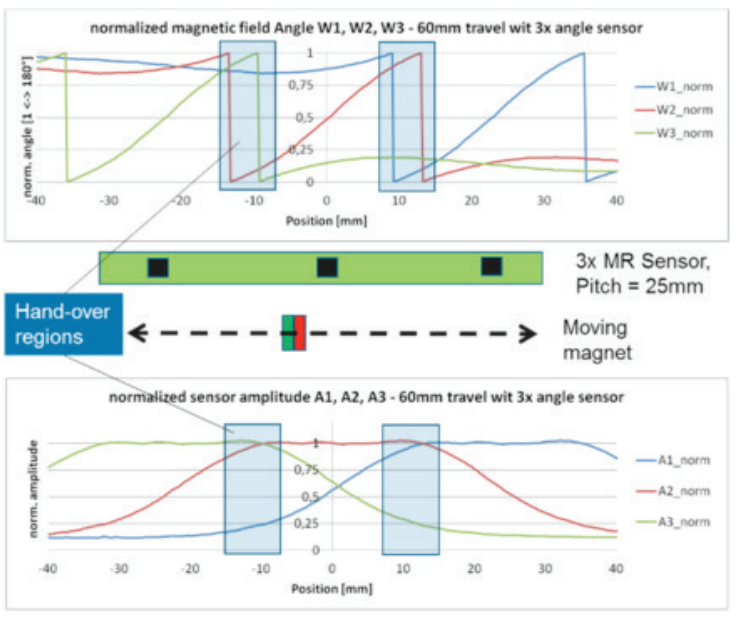

Figure 9 absolute position determination with an array on sensors

As an absolute measurement needs to work with a one shot capture of the sensor signals when measuring the field vector information of the sensed magnet - robust criterias are needed to determine the magnet position correctly. Therefore the algorithm can be separated into several steps:

1. Initially the sensor signals need to be offset free. To cancel out the offsets a left-right movement of the magnet along the whole sensor chain is performed. During this, diagnostic data of the sensor array is recorded and all needed offset canceling parameters are calculated. This could be done within an end-of-line test and represents a calibration of the sensor system to the used magnet.

2. When using the so called amplitude criteria, those sensors, which are directly affected by the magnet are filtered out. This will lead to a raw position information of the magnet.

3. Now the next adjacent sensors at the calculated raw position are taken into account to determine a medium accurate position information of the magnet. As result, it is known, which sensor of the chain has the shortest distance to the magnet.

4. A last step is interpolating a previous calibrated Look-Up-Table (LUT) to link the measured angle information of the sensor to a fine position result.

The position accuracy of such an array based sensor system is mainly driven by the accuracy of the 1:1 relationship between sensor and magnet. So it is directly connected to the filed distribution of the magnet and the corresponding lookup table mentioned above. A typical arrangement with AMR sensors aligned in a pitch of $20 \mathrm{~mm}$ leads to position accuracies of $+/-0,5 \mathrm{~mm}$ in a temperature range of $-40^{\circ} \mathrm{C}$ to $+125^{\circ} \mathrm{C}$ over $60 \mathrm{~mm}$ as shown in the example in figure 10:

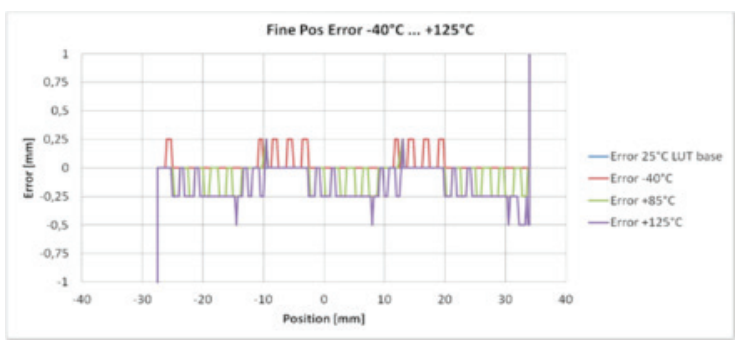

Figure 10 The position accuracy of sensor array consisting of 3 AMR angle sensors, aligned in pitch of $20 \mathrm{~mm}$ in a temperature range of $\left[-40^{\circ} \mathrm{C} \quad \ldots\right.$ $\left.+125^{\circ} \mathrm{C}\right]$

\section{Covering longer distances with $\mathbf{n}$ sensors}

Customer demands are widely diversified when talking about distance, resolution, accuracy, magnet types \& geometries etc.

To cover the majority of these demands, a linear position sensor based on the described principle can be adapted to different lengths. When using a chain of more than 3 sensors, the described algorithm needs to be enhanced. Those 3 sensors, which are directly adjacent to 
the magnet as shown in figure 11 are filtered out by a preceded and modified second amplitude criteria.

A special case is found when the magnet is at the boundary range of the sensor chain, where just the last two sensors are taken into account. The sensor system design enables this step by placing the used sensors in a pitch where a one shot measurement allows the filtering of these sensors directly.

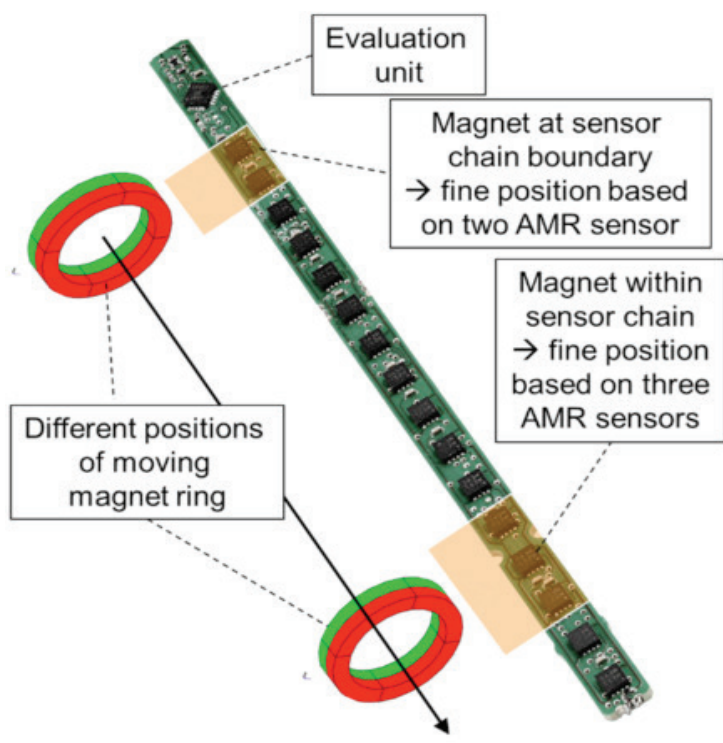

Figure 11 A linear AMR sensor array consisting of 16 sensor, filtering of the most adjacent sensors to the magnet by using the amplitude-criteria

Once the three (or two) nearest sensors are chosen, the algorithm could start in determining the magnet position following the steps shown above.

As the calculation of the fine position is lead back to the determination of the most adjacent sensor (or in a special case the two most adjacent sensors, when the magnet position is found in the hand-over region) to the magnet, the calculation is broken down to a single sensor measurement. Therefore looking from the percentage of the covered position range, the overall position accuracy results in more accurate measurement systems, the longer the covered distance will be.

\section{Outlook}

Actually the shown sensor systems are based on single AMR sensor components, coupled together to establish an array-chain architecture. As the described sensor approach has to work with a bigger number of sensor nodes, future developments will need to find an optimum of the used magnet geometry, the number of needed sensors together with an optimum alignment of these sensors to realize a precise and economic measurement over a certain distance.

As many measurement tasks have different accuracy demands [6] on several sections of the measurement range, a higher density of sensor nodes at these areas could help to enhance the system accuracy punctually. Here an average of the involved sensors will lead to higher position accuracies.

\section{References}

[1] U. Dibbern: Magnetic field sensors using the magnetoresistive Effect, Sensors and Avtuators, 10, pp 127-140, 1986

[2] T.R. McGuire, R.T. Potter: Anisotropic magnetoresistance in ferromagnetic $3 d$ alloys, IEEE Transactions on Magnetics, Vol. Mag-11, No- 4, 7/1975

[3] Magnetfabrik Bonn, Applications Brief 1/2008: The Effects of Temperature on Permanent Magnets

http://www.magnetfabrik.de/magnetfabrik_en/up loads/1237295649Praxis 0108 eng final.pdf (26.02.2016)

[4] S.Shtrikman, D.R. Smith: Analytical formulas for the unshielded magnetoresistive head, IEEE TRANSACTIONS ON MAGNETICS. VOL 32, NO 3. MAY 1996

[5] A.Voss, A.Meisenberg, R.Pieper, A.Bartos: Absolute Positionsbestimmung mit magnetoresistiven Sensoren, Mikrosystemtechnik Kongress 2013 pp 215 - 218, ISBN 9783-8007-3555-6

[6] A.Voss, A.Meisenberg, A.Bartos: High definition level transducer based on magnetoresistive sensors , Proceedings of Sensors and Measuring Systems 2014; 17. ITG/GMA Symposium; ISBN 978-3-8007-3622-5 